\title{
Breakdown phenomenon in insulators of submarine power cables
}

\author{
D. Malec and R. Essolbi \\ Laboratoire de Génie Electrique (CNRS UA 304), Université Paul Sabatier, 118 route de \\ Narbonne, 31062 Toulouse Cedex, France
}

(Received 25 May 1992, revised 19 February 1993, accepted 16 March 1993)

\begin{abstract}
Dielectric characteristics of polyethylenes used for the cable insulation under submarine working conditions (temperature and pressure) have been investigated by the authors. From experimental results, a modelling of the short time breakdown was proposed by numerically solving the fundamental heat balance equation. Electromechanical effect has been also taken into account in this model. Theoretical results are in good agreement with observed data.
\end{abstract}

\section{Introduction.}

Submarine D.C. power cables with extruded synthetic insulation have the advantages of simplifying the cable-laying and reducing the risks of deterioration during installations at great depth. The cable is thereafter submitted to a hydrostatic pressure of one bar for every ten meters of depth. Consequently, it is necessary to know the dielectric characteristics of the polyethylenes used for the cable insulation under their working conditions of temperature ( 30 to $70{ }^{\circ} \mathrm{C}$ ) and pressure $(50$ to 500 bar).

In this paper, the dielectrical properties of these materials were investigated by applying high electrical fields to measure the conductivity, and destructive stresses to measure the breakdown field. The purpose is to know first how these characteristics vary and second how the breakdown phenomenon occurs in these insulators under the same conditions.

\section{Experimental apparatus.}

The experimental apparatus (Fig. 1) is composed of a high pressure bomb $\left(9500 \mathrm{~cm}^{3}\right)$, a measuring cell and a pressure generator (1 to 1500 bar) [1]. The measuring cell consists of stainless steel electrodes with Rogowsky profile $(\phi=14 \mathrm{~mm})$ switching by high voltage relays (Fig. 2). It is used to study eight $100 \mu \mathrm{m}$ thick samples of High Density Polyethylene (HDPE) and Cross-linked Polyethylene (XLPE) directly cut into high voltage cables. The pressure is obtained by a two-stages diaphragm compressor using gaseous nitrogen as 


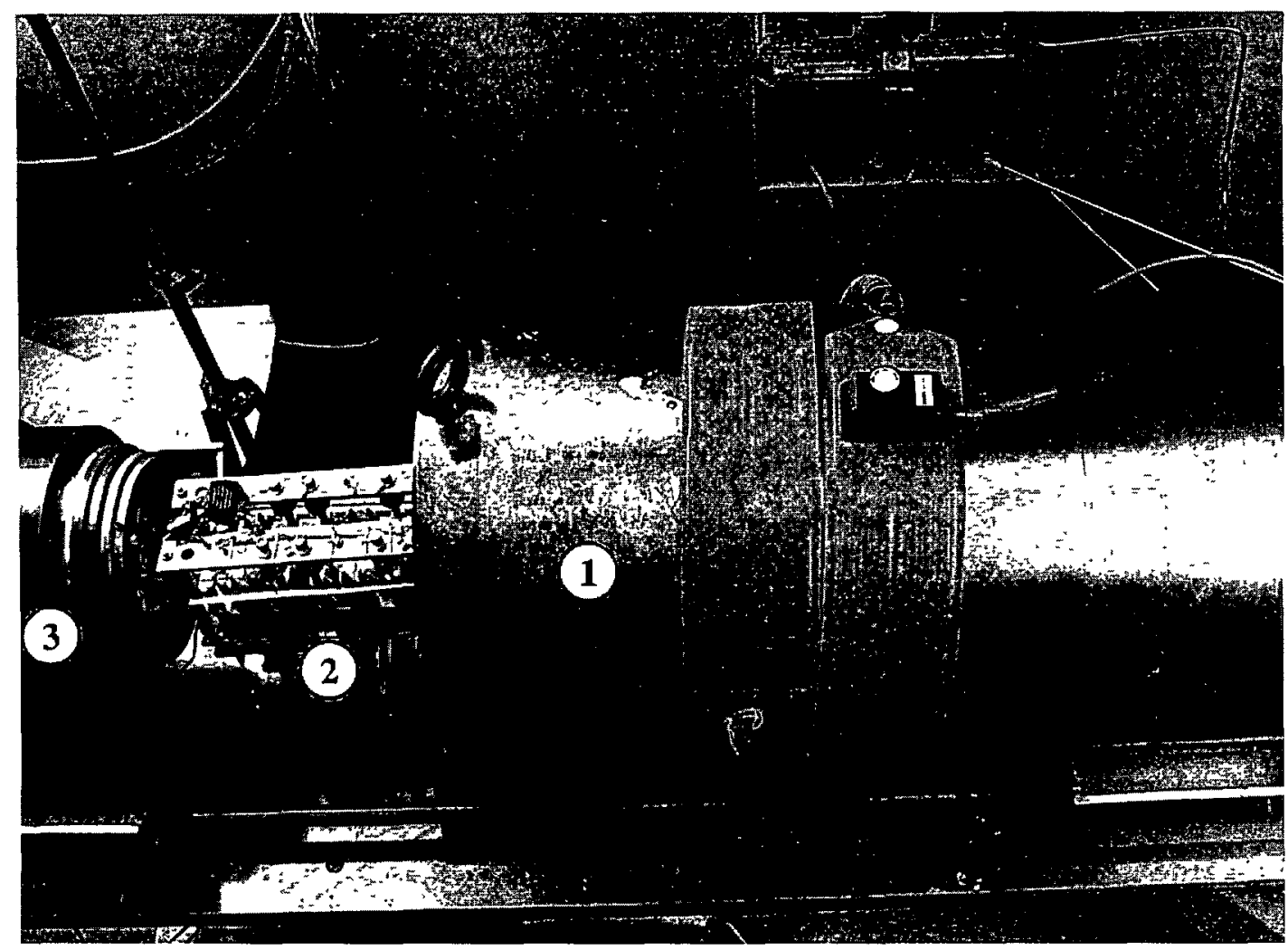

Fig. 1. - Experimental apparatus. $1=$ high pressure bomb. $2=$ measuring cell. $3=$ obturator.

transmitting fluid. The desired temperature is established by heating resistors with a stability of better than $0.5^{\circ} \mathrm{C}$. High voltage is produced by a $100 \mathrm{kV} \mathrm{D.C}$. generator. The conduction current is measured by an electrometer with a resolution of $10^{-14} \mathrm{~A}$ and the breakdown field is detected by an electro-optical circuit. Two special electrical leads having very high leakage resistance, excellent mechanical and thermal properties are used for the minimum current level measurement and the applied voltage up to $80 \mathrm{kV}$ on D.C.

\section{Experimental results and modelling.}

3.1 Conductivity measurements. - The polarization of the samples is maintained up to the steady-state conduction current. The electrometer is connected to a micro-computer in which the current versus time is stored and directly plotted with a recorder. The variations of the conduction current versus the applied field, the temperature and the pressure are summarized in figures 3 and 4 .

Results have shown that for all polyethylenes, the conduction current increases with the temperature. A decreasing and an increasing current with pressure are observed with HDPE and XLPE samples respectively.

3.2 Conductivity MODELling. - The assignement of charge carriers are difficult problems for most insulating polymers. There are only a small number of them whose carriers are well known. Between the methods applicable to prove ionic conduction, the change in free volume 


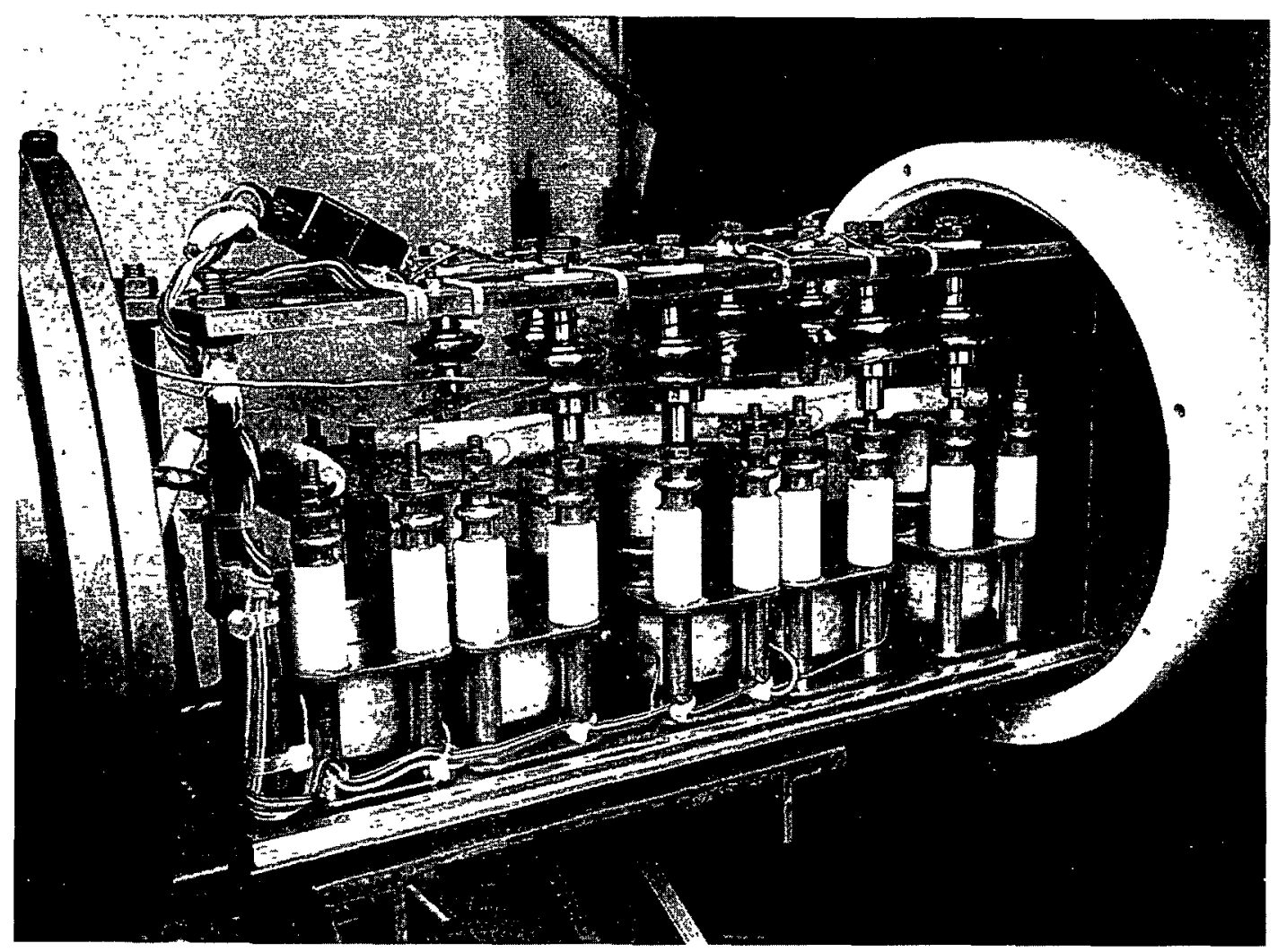

Fig. 2. - Measuring cell equipped with Rogowsky profile electrodes and high voltage relays.

caused by pressure is one of them [2-4]. Consequently, the decrease of HDPE current versus pressure could be explained by an ionic conduction model assuming that ions are predominant. An increase in pressure decreases the free volume $V_{\mathrm{f}}$ and consequently the ionic conductivity resulting of the motion of ions between two equilibrium positions separated by a jump distance $\lambda$. The model chosen was the one developed by Miyamoto et al. [5] in which we have introduced the new parameter pressure $P$. According to our hypothesis, the current density could be expressed by :

$$
J(P, T, F)=J_{0} \lambda(P) \cdot \exp \left(-\frac{\gamma V_{1}}{V_{\mathrm{f}}}(P)-\frac{E_{\mathrm{J}}+\frac{W}{2 \varepsilon}}{k T}\right) \cdot \sinh \left(\frac{q \lambda(P) F}{2 k T}\right)
$$

where $J_{0}$ is a constant, $\varepsilon$ the dielectric constant, $k$ the Boltzmann's constant, $q$ the charge, $F$ the applied field and $T$ the absolute temperature. In this equation, the term $\exp \left(-\gamma V_{\mathrm{i}} / V_{\mathrm{f}}\right)$ expresses the probability for an ion of finding a hole whose size is greater than a critical volume $V_{1}$ ( $\gamma$ is a numerical factor to correct the overlap of free volume). Furthermore, the probability for jump by surmounting the potential barrier $E_{\mathrm{j}}$ and the dissociation energy $W$ is given by $\exp \left(-\frac{E_{\mathrm{J}}+\frac{W}{2 \varepsilon}}{k T}\right)$, according to the theory of rate processes [6] and the ionic 


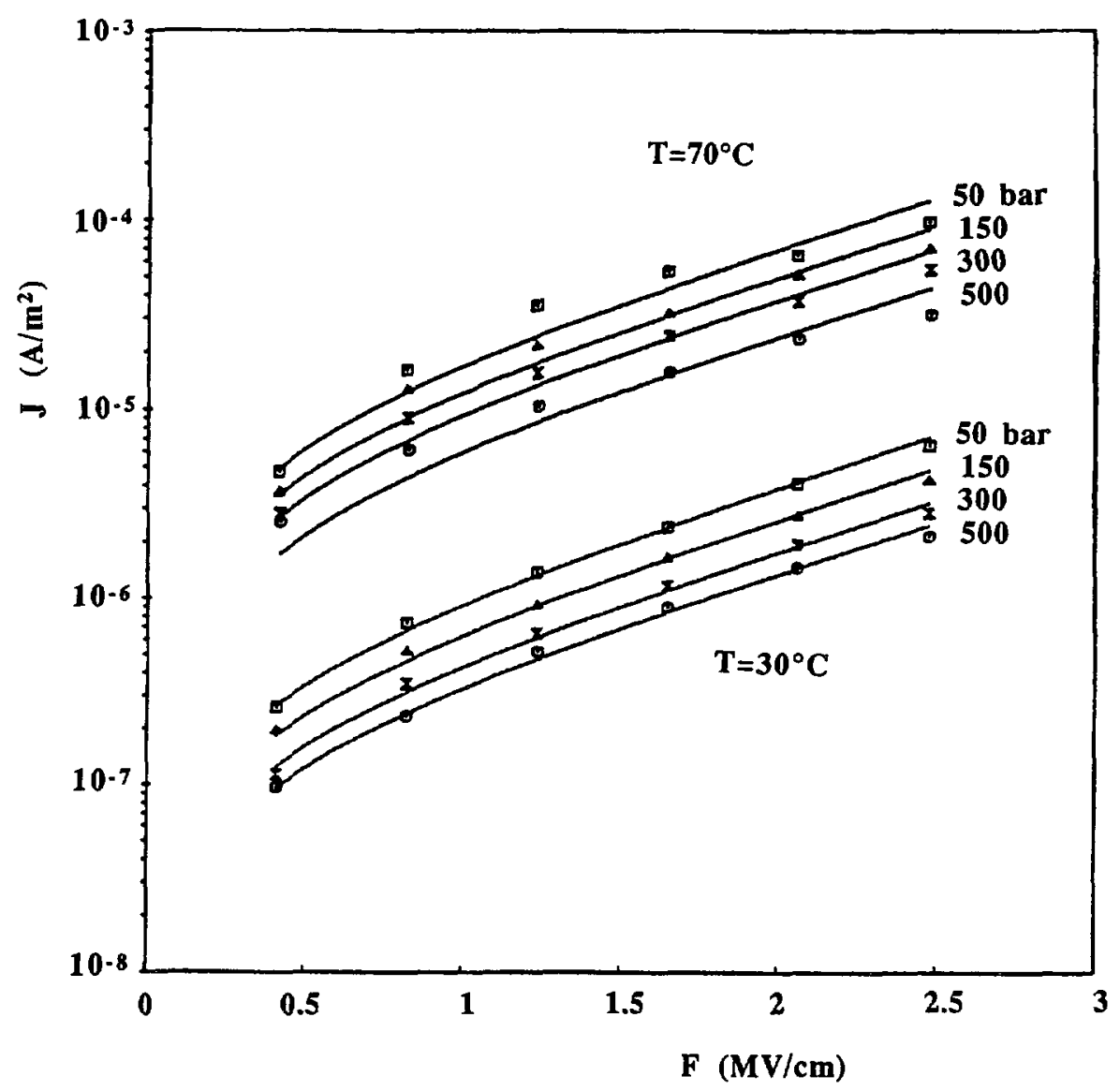

Fig. 3. $-J(F)$ characteristics of HDPE for different temperatures and pressures.

dissociation theory [7] respectively. Using the curve-fitting method based on an optimization calculation [8], this theoretical model of conduction was applied to our experimental results.

Experimental data (points) and theoretical curves (straight lines) are plotted in figure 3. Parameters used for $P=50$ bars are :

$$
\lambda=7 \AA . \quad \gamma V_{\mathrm{i}} / V_{\mathrm{f}}=1.8, \quad E_{\mathrm{J}}+W / 2 \varepsilon=0.62 \mathrm{eV} \quad \text { and } \quad J_{0}=10^{14} \mathrm{~A} / \mathrm{m}^{3}
$$

From these results, it seems that the ionic conduction, due to the existence of impurities, additives or extraneous ions, could be used for explaining the variation of the HDPE current density versus applied field, temperature and pressure.

Concerning XLPE, we assume that an increase in pressure leads to an increase in electron mobility by decreasing the inter-site distances. Electron injection process could appear in XLPE, conditioned by the storage of a space charge accumulated near the cathode, as suggested by spatial charge distribution measurements $[9,10]$. So that, electronic conduction could be explained in terms of Schottky-thermionic injection at the cathode and Poole-Frenkel detrapping mechanism in the bulk of the sample [11]. Schottky-emission effect becomes more important at high electric field level, since the curve of $\log J$ versus $F^{1 / 2}$ is almost a straight line. The current density variations could be expressed by [12] :

$$
J=A \cdot T^{2} \cdot \exp (-(\phi-\Delta \phi) / k \cdot T)
$$




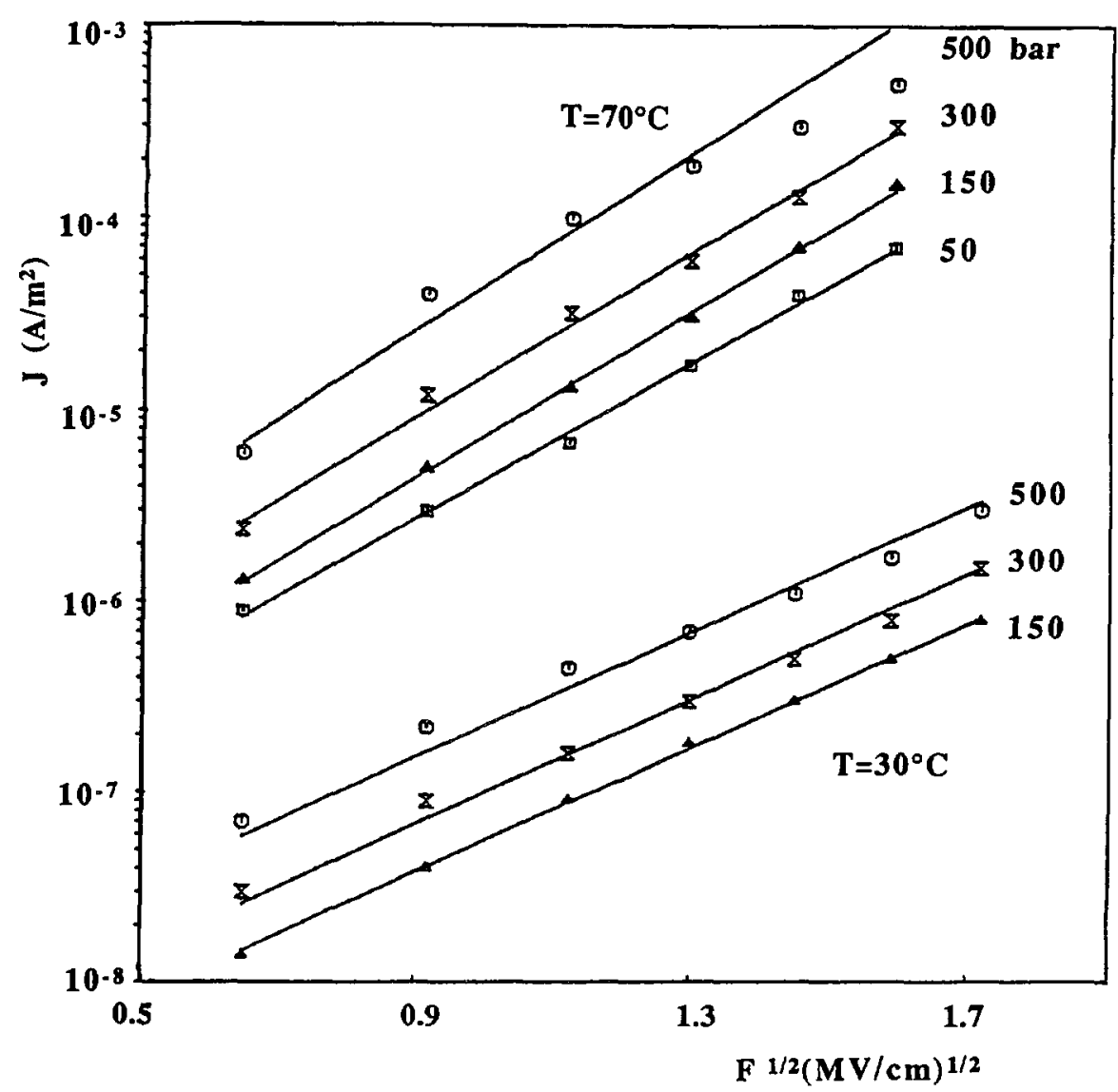

Fig. 4. $-J(F)$ characteristics of XLPE for different temperatures and pressures.

where $A$ and $k$ are the Richardson and Boltzmann's constants respectively; $\phi$ and $\Delta \phi$ represent the barrier height and its lowering due to the electric field respectively. Experimental and theoretical results are shown in figure 4 with $\phi=1.2 \mathrm{eV}$.

3.3 BREAKDOWN FIELD MEASUREMENTS. - A ramp voltage with a rise rate of $5 \mathrm{kV} / \mathrm{s}$ is applied to samples up to the appearing of the breakdown process. The breakdown field values so obtained are analyzed by the Weibull's statistical theory [13], because of the random distribution of defaults existing in the material. The breakdown field is defined as the probable value corresponding to $63.2 \%$ of the breakdown probability of failure in the Weibull probability diagram (Fig. 5). The breakdown field of HDPE and XLPE increases with pressure up to $20 \%$ and decreases with temperature up to $10 \%$ (Figs. 7 and 8 ).

3.4 MODELLING OF THE DIELECTRIC BREAKDOWN. - There are three possible mechanisms to explain the negative temperature dependence of the breakdown field $F_{0}$. the thermal breakdown, the electronic breakdown in amorphous materials presented by Fröhlich and the electromechanical breakdown [14]. Fröhlich's theory cannot explain the dependence of $F_{0}$ on the voltage rising rate observed in polyethylene [15]. Following our hypothesis, the breakdown field variations with pressure and temperature may be explained by the thermal breakdown theory. Because of the existence of the conductivity in the material, the applied 


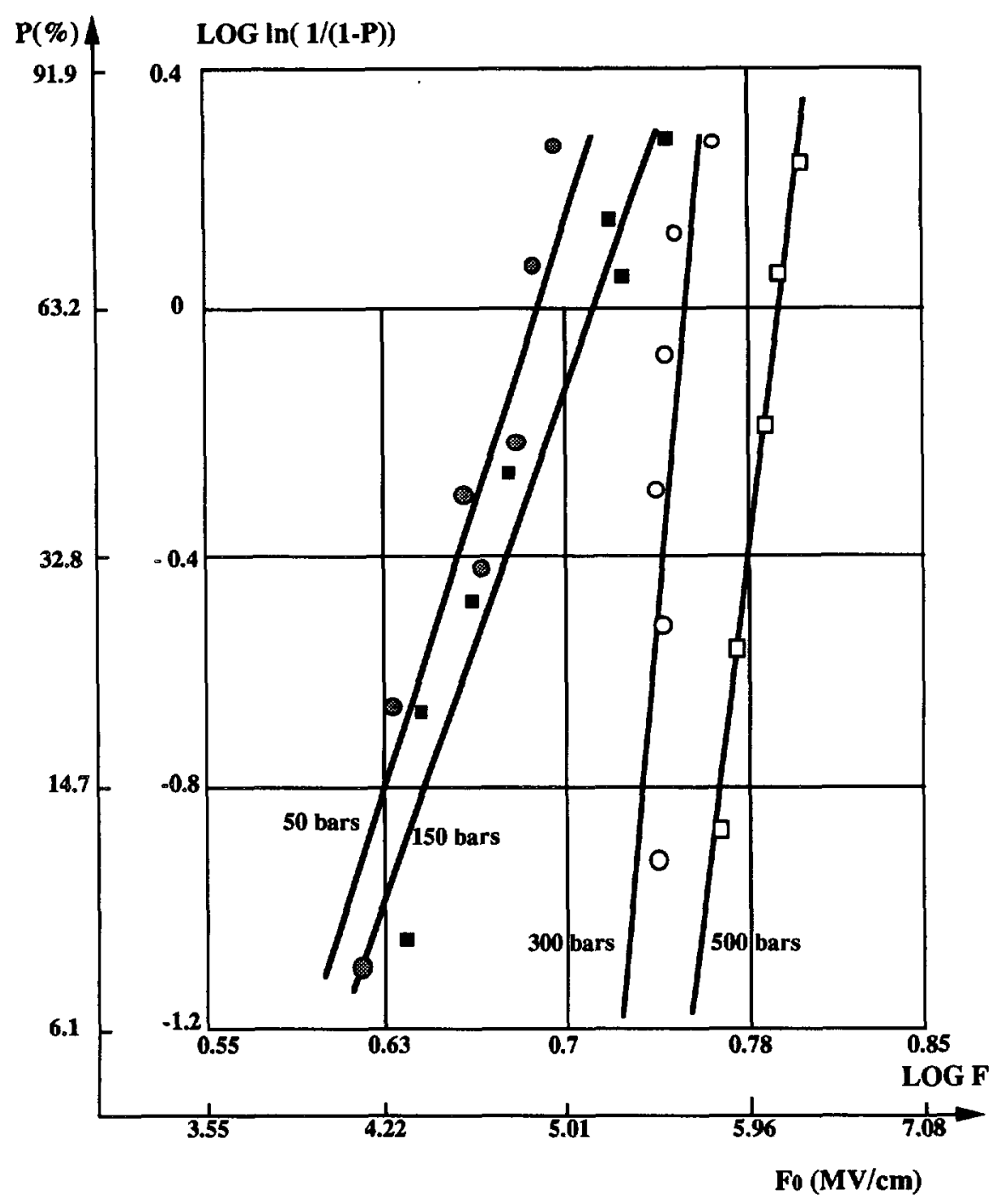

Fig. 5. - Weibull's diagram of XLPE at $T_{0}=70^{\circ} \mathrm{C}$ for different pressures.

field effect is no induce an increase of the temperature in the sample up to its melting point $\left(130^{\circ} \mathrm{C}\right.$ for HDPE and $105^{\circ} \mathrm{C}$ for XLPE). This thermal breakdown process may be analyzed in light of the equilibrium between Joule heating due to the electrical conduction and its dissipation due to the thermal conduction $[14,16]$. The fundamental equation for the thermal breakdown, or the energy balance equation, following the energy balance equation, can be expressed in one dimension as follows (width and breadth much larger than thickness):

$$
C_{\mathrm{v}} \cdot(\partial T / \partial t)-K \cdot\left(\partial^{2} T / \partial x^{2}\right)=\sigma \cdot F^{2}
$$

The first term represents the increase of temperature versus time $\left(C_{\mathrm{v}}=\right.$ specific heat per unit volume), the second the thermal dissipation ( $K=$ thermal conductivity) and $\sigma . F^{2}$ the rate of energy gain from the applied field $F$ ( $\sigma=$ electrical conductivity). As Hikita [17], we can 


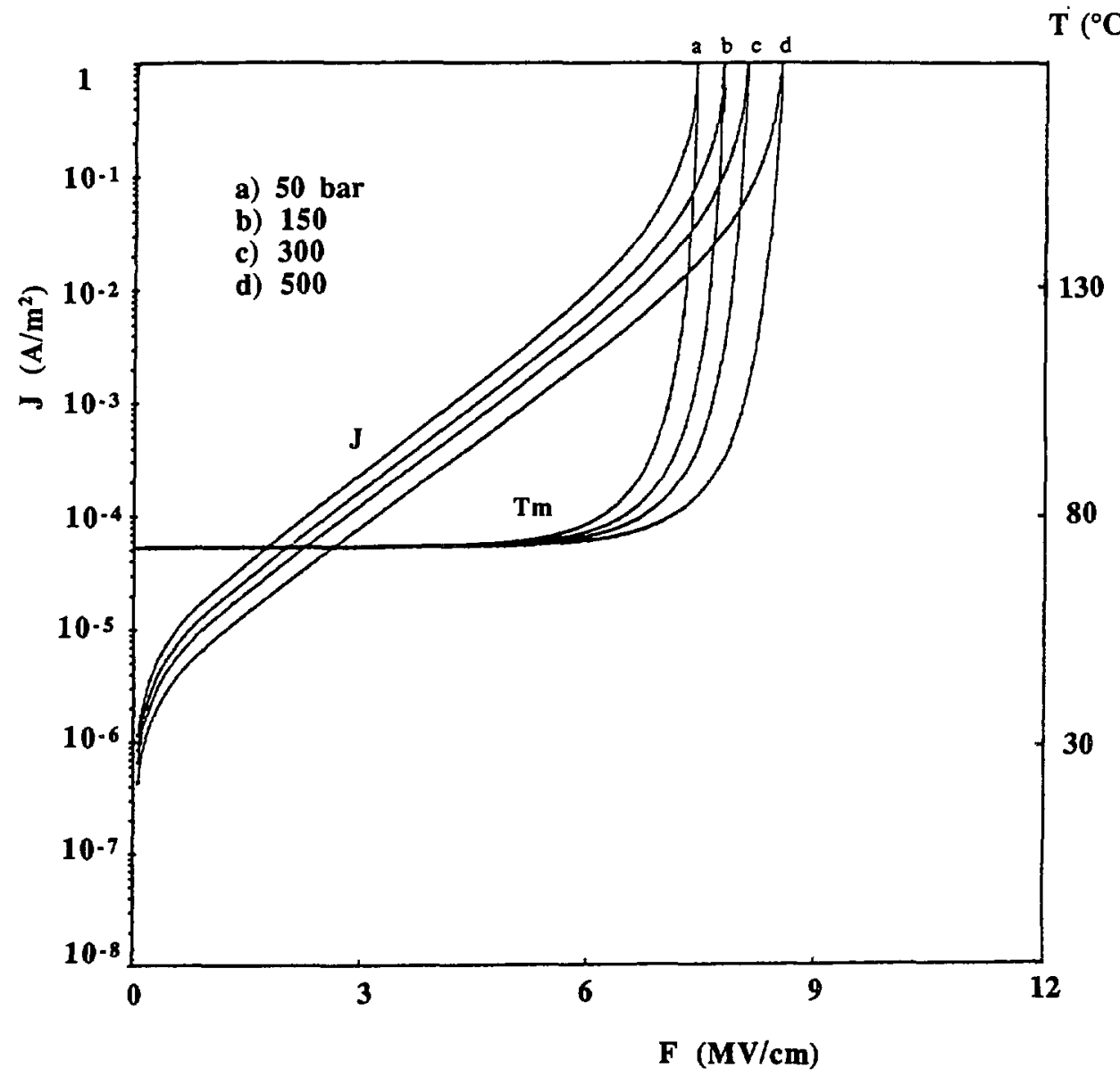

Fig. 6. - Theoretical current density $J$ and temperature $T_{\mathrm{m}}$ in the central part of the bulk (HDPE sample) versus field for different pressures. $T_{0}=70^{\circ} \mathrm{C}, C_{\mathrm{y}}=2.2 \times 10^{6} \mathrm{~J} / \mathrm{m}^{3} \mathrm{~K}, K=0.35 \mathrm{~W} / \mathrm{mK}$ and $H=$ $0.1 \mathrm{~W} / \mathrm{m}^{2} \mathrm{~K}$.

consider our conduction model as valid for applied field higher than $2.5 \mathrm{MV} / \mathrm{cm}$. Indeed, he showed that the fitting of experimental data at high field on polyethylene samples agree well with the behavior of the current in the vicinity of breakdown.

Thermal equation could be resolved by the numerical method based on the transformation of the above differential terms into finite differences [18], with the boundary conditions :

- $T=T_{\max }$ for $x=0$ (midplane)

- $H\left(T-T_{0}\right)$ for $x=\frac{ \pm}{-} d / 2$ (edges of the sample), with $H=$ heat transfer coefficient and

$T_{0}=$ room temperature. It's assumed than the heat dissipation from the dielectric surface to the surrounding obeys Newton's law of cooling.

Figures 6 and 7 show some results obtained for HDPE. From the qualitative point of view, a good agreement is observed by comparing theoretical and experimental results of breakdown field. If we consider that the testing electrodes were not mechanically attached, the electrostatic force caused by the applied field must be taken into account. The mechanical strength of polyethylene can be estimated from the variation of its Young's modulus versus temperature [17, 20] and hydrostatic pressure [21]. 


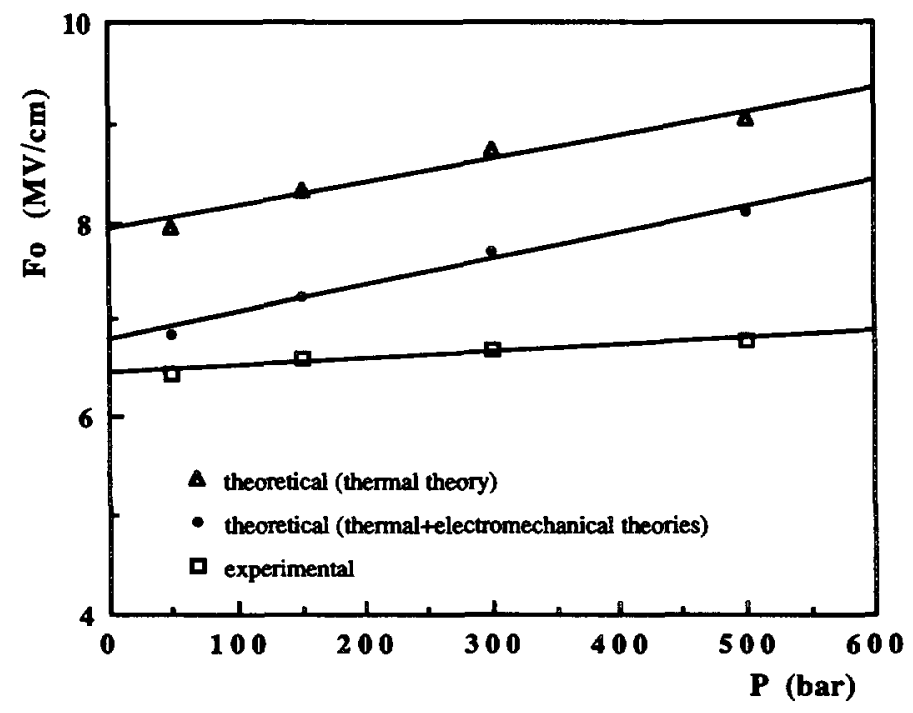

Fig. 7. - Theoretical and experimental characteristics of the breakdown field $F_{0}$ of HDPE versus pressure $P\left(T_{0}=70^{\circ} \mathrm{C}\right)$.

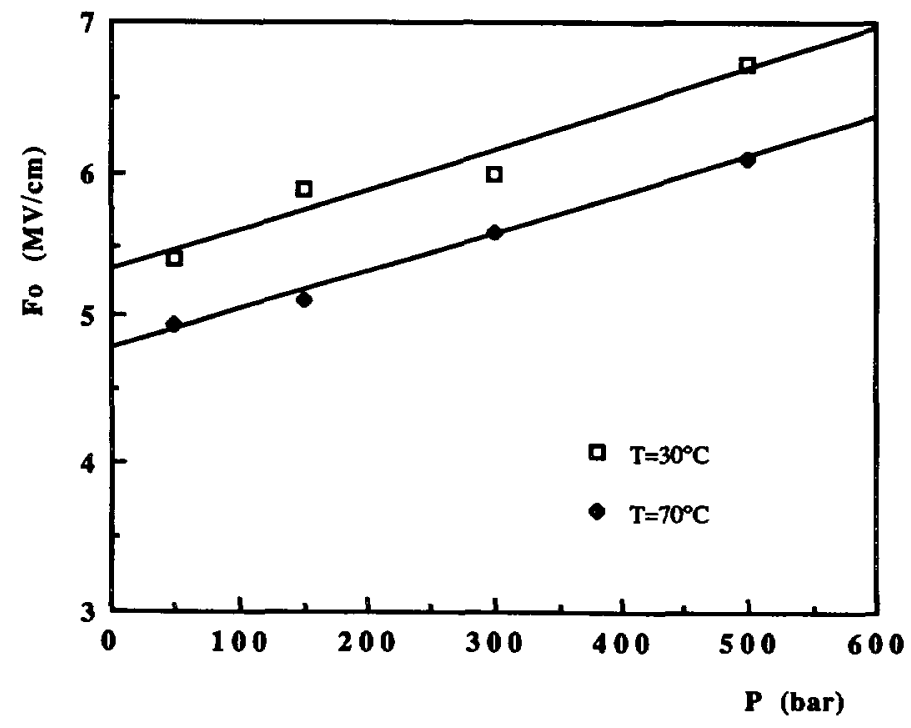

Fig. 8. - Experimental characteristics of $F_{0}$ versus pressure $P$ and temperature $T_{0}$ for XLPE.

From these results, it seems that the thermal breakdown theory with the contribution of electromechanical effect can explain only the breakdown field variations of HDPE with pressure and temperature. In general, the thermal breakdown field decreases with the increasing conductivity. The results of XLPE seem to be completely opposite to this prediction. In order to elucidate this breakdown mechanism, it is required to consider other interpretation for the conductivity measurement, such as space charge with the existence of a distortion in the internal field. Moreover, other interpretation for the measurement of the 
breakdown field could be considered since it seems to be caused by local filamentary heat generation instead of whole area heat generation as assumed by this model.

\section{Conclusion.}

From the experimental point of view, this study has shown that the pressure affecting the working conditions of submarine power cables might give a better dielectrical behavior of insulators with increasing hydrostatic pressure. These results agree with those obtained with the life-time tests [22].

From the theoretical point of view, the new parameter pressure was introduced in the study of the breakdown and the conductivity phenomena in solid insulators. The breakdown mechanism occurring in HDPE samples with a DC ramp voltage was explained in terms of thermal and mechanical processes by using the experimental results on high field conduction and Young's modulus. This study have shown that it is difficult to specify the breakdown phenomenon in XLPE by considering the Schottky-emission as predominant in high field conductivity. Further investigations are necessary, for example by considering internal field distribution established by the presence of a space charge in the material.

\section{References}

[1] Hoang-The-Giam et al, Revue Générale de l'Electricté, n 11 (1989) 19-22.

[2] SAITo S., 1st Symp. Elect. Insul. Material., IEE Jph (1968).

[3] BAIRD M. E., Electrical properties of polymeric materials, The Plastics Institute (London, 1973).

[4] IEDA M., International Conference on Conduction and Breakdown in Solid Dielectrics (Toulouse, 1983).

[5] Miyamoto T., Shibayama K., J. Appl. Phys. 44 (1973) 5372-5376.

[6] Glasstone S., Eyring H., The theory of rate processes (McGraw-Hill, New York, 1947).

[7] Barker R. E., Thomas C. R.. J. Appl. Phys. n 35 (1964) p. 3203.

[8] Moukarzel I., Thesis (Toulouse University, 1987).

[9] Li Ying et al., 3rd International Conference on Properties and Applications of Dielectric (Tokyo, 1991).

[10] Fukunaga K. et al., 3rd International Conference on Polymer insulated power cables (Versailles, 1991).

[11] Pulfrey D. L. et al., J. Appl. Phys. 41 (1970) 2838-2843.

[12] LAMB D. R.. Electrical Conduction mechanisms in thin films (Worsnop editor, 1967).

[13] Weibull W., J. Appl. Mech. 18 (1951) 293-297.

[14] O'DWYER J. J., The theory of electrical conduction and breakdown in solid dielectrics (Clarendon Press, Oxford, 1973).

[15] GUÉRIN P.. Thesis (Toulouse University, 1984).

[16] Wagner K. W., Trans. AIEE, n 41 (1922) 288.

[17] Hikita M. et al., Jpn J. Appl. Phys. 24 (1985) 988-996.

[18] Nougier J., Méthode de calcul numérique (Edition Masson, Paris, 1983).

[19] STARK K. H., Garton C. G., Nature 176 (1955) 1225-1226.

[20] HikiTA M., Thesis. Nagoya University (Japan, 1982).

[21] Ainbinder S. B., et al, Mekhanika \& Polimerov. n 1 (1965) 65.

[22] Benlizidia F., Thesis (Toulouse University, 1992). 